\title{
NoD0S(3) Calling!! Propuesta de trazos, huellas y recorridos como resultado de una residencia artística online
}

\author{
NoDOS (3) Calling!! Proposal of lines, footprints and routes as a \\ result of an online artistic residency
}

Elia Torrecilla

elia.torrecilla@gmail.com

Cristina Ghetti

cristighetti@gmail.com

Universidad Miguel Hernández, Elche (España)
Recibido 04/09/2020 Revisado 10/10/2020

Aceptado 12/10/2020 Publicado 31/07/2020

\section{Resumen:}

En el presente fotoensayo se comparte un proyecto artístico intermedia, realizado en The Spectacular House, una plataforma de divulgación de arte y pensamiento, comisariada por Sofia Caetano y establecida en Pittsburgh (USA) y Ponta Delgada (Azores). Su labor se muestra en una página web creada con el objetivo de promover el arte y la cultura de manera virtual a escala global. Dentro de esta plataforma, se encuentra "Gate", una puerta que se abre hacia una galería de arte online donde se alojan proyectos site specific y proyectos generados en Internet como parte del proceso desarrollado en un entorno de residencia artística online. Y es dentro de este contexto donde el Grupo NoDOS(3), formado por Cristina Ghetti y Elia Torrecilla, desarrolló un proyecto durante dos semanas. El resultado final es una pieza digital, con una fuerte presencia de lo sonoro, donde las interacciones del usuario, las experiencias de desplazamientos, hipervínculos, vídeos, fotos y recorridos múltiples permiten visualizar un trabajo de naturaleza híbrida, de una manera diferente y creativa.

En este fotoensayo, se propone una visualización del proyecto a través de imágenes que muestran nuestro proceso de trabajo y que permiten al lector atravesar la pantalla a través del hipervínculo. De este modo, el visitante podrá participar de las investigaciones que NoDOS(3) llevamos a cabo, y que están basadas en el uso del cuerpo y la tecnología en el espacio (físico, virtual e híbrido) a través de la performance. En definitiva, ofrecemos la posibilidad de que el visitante realice un recorrido de manera lúdica e interactiva a través de un fotoensayo a golpe de "click".

Sugerencias para citar este artículo,

Torrecilla, Elia; Ghetti, Cristina, (2020). NoDOS (3) Calling!! Propuesta de trazos, huellas y recorridos como resultado de una residencia artística online. Tercio Creciente (Monográfico extraordinario II), págs. 137-153, https://dx.doi.org/10.17561/rtc.extra2.5726

TORRECILLA, ELIA; GHETTI, CRISTINA. NoDOS (3) Calling!! Propuesta de trazos, huellas y recorridos como resultado de una residencia artística online. Tercio Creciente (Monográfico extraordinario II), octubre 2020, pp. 137-153, https://dx.doi.org/10.17561/rtc.extra2.5726 


\section{Abstract:}

In this photo essay an intermediate artistic project is shared, carried out at The Spectacular House, a platform for the dissemination of art and thought, curated by Sofia Caetano and established in Pittsburgh (USA) and Ponta Delgada (Azores). His work is displayed on a website created with the aim of promoting art and culture virtually on a global scale. Within this platform, there is "Gate", a door that opens to an online art gallery where site specific projects and projects generated on the Internet are hosted as part of the process developed in an online artistic residency environment. And it is within this context that the NoDOS Group (3), made up of Cristina Ghetti and Elia Torrecilla, developed a project for two weeks. The end result is a digital piece, with a strong presence of sound, where user interactions, travel experiences, hyperlinks, videos, photos and multiple tours allow visualizing a work of a hybrid nature, in a different and creative way.

In this photo essay, a visualization of the project is proposed through images that show our work process and that allow the reader to cross the screen through the hyperlink. In this way, the visitor will be able to participate in the investigations that NoDOS (3) carry out, and that are based on the use of the body and technology in space (physical, virtual and hybrid) through performance. In short, we offer the possibility for the visitor to take a tour in a fun and interactive way through a photo-essay at the click of a button.

Palabras Clave: Arte, performance, virtual, híbrido, residencia artística.

Key words: Art, performance, virtual, hybrid, artistic residency.

Sugerencias para citar este artículo,

Torrecilla, Elia; Ghetti, Cristina, (2020). NoDOS (3) Calling!! Propuesta de trazos, huellas y recorridos como resultado de una residencia artística online. Tercio Creciente (Monográfico extraordinario II), págs. 137-153, https://dx.doi.org/10.17561/rtc.extra2.5726

TORRECILLA, ELIA; GHETTI, CRISTINA. NoDOS (3) Calling!! Propuesta de trazos, huellas y recorridos como resultado de una residencia artística online. Tercio Creciente (Monográfico extraordinario II), octubre 2020, pp. 137-153, https://dx.doi.org/10.17561/rtc.extra2.5726 


\section{Introducción: ¿Qué es The Spectacular House?}

The Spectacular House es una plataforma de divulgación de arte y pensamiento basada en Pittsburgh USA y Ponta Delgada Azores, cuya labor se vuelca en una página web con la premisa de compartir y divulgar arte y pensamiento artístico de manera virtual a escala global.

\section{Perfil de los Invitados}

Esta residencia funciona por invitación de la directora del espacio y comisaria Sofia Caetano. El perfil de invitados son artistas visuales o sonoros internacionales cuyo trabajo puede ser visualizado vía online. En este enlace se puede acceder al archivo del los diferentes artistas que realizaron las residencia artística online: https://www.thespectacularhouse.com/gategallery-residency-archives

\section{Idea de nuestra participación}

NoDOS(3) Calling! Es una pieza realizada y concebida para ser vista online. La pieza es una presentación de nuestro grupo artístico interdisciplinar "NoDOS(3)" formado por Cristina Ghetti y Elia Torrecilla, que está abierto a la colaboración con otrxs artistas.

Esta propuesta ofrece la posibilidad de recorrer nuestros trabajos de una manera lúdica e interactiva.

Dentro de la página del proyecto, que funciona como un espacio de arte y producción, "GATE" es la galería de arte, donde se combinan proyectos site specific y proyectos generados en internet y desarrollados dentro de un entorno de residencia online.

NoDOS(3) fue invitado a realizar su obra durante un periodo de trabajo online de dos semanas (residencia virtual), durante el cual se realizaron intercambios creativos con la comisaria. El resultado final fue expuesto en la página web de the Spectacular House: https://www.thespectacularhouse.com/nodos-3

El día de la presentación, se realizó un encuentro virtual, a modo de inauguración, en el que fuimos invitadas a presentar nuestro trabajo vía streaming para intercambiar ideas sobre nuestros proyectos en particular, y también para reflexionar sobre el desarrollo de proyectos en estos tiempos de confinamiento.

La pieza fue expuesta en GATE de The Spectacular House durante un periodo de dos meses. Transcurrido ese periodo, nuestra página pasa a formar parte del archivo de artistas residentes. 
Aprovechando la potencialidad del medio (página web), decidimos adaptar nuestro trabajo y dotarlo de interactividad, para que el usuario pudiera transitar por las acciones realizadas y su registro, dejándose llevar por el hipertexto.

\section{Desarrollo-estructura del recorrido visual}

A partir de un portal con una imagen de las performers, se puede clickar sobre diez iconos que remiten a cada una de las acciones realizadas por el grupo. Al entrar en cada acción, el usuario accede a una descripción de la misma junto con la posibilidad de participar en un pequeño juego interactivo visual y sonoro.

Además de los registros de nuestras acciones (fotografía, vídeos, textos...), ofrecemos la experiencia de que el navegante visite aquellos espacios donde fueron realizadas dichas acciones. A través de Google Street View, el navegante puede pasear por las calles de un espacio virtual que representa el espacio físico.

Asimismo, como parte del juego visual, diseñamos unos gif que muestran una experiencia estética de la base de nuestras acciones: la línea. 
ISSN: 2340-9096

https://dx.doi.org/10.17561/rtc.extra2.5726

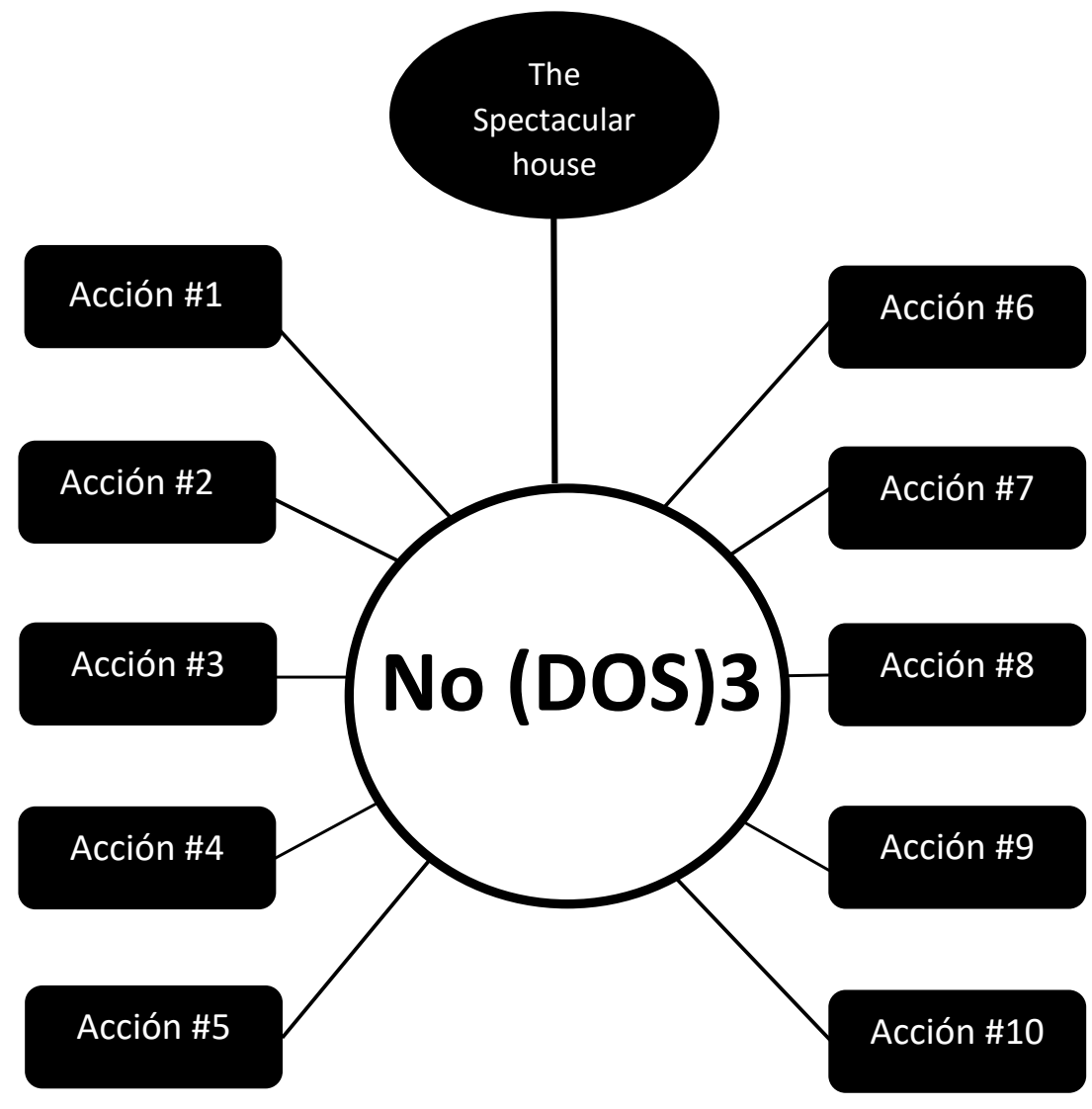

NoDOS(3) (2020). Estructura de la página web. Fuente: las autoras 
Revista de Estudios en Sociedad, Artes y Gestión Cultural

ISSN: 2340-9096

https://dx.doi.org/10.17561/rtc.extra2.5726

NoDOS(3)
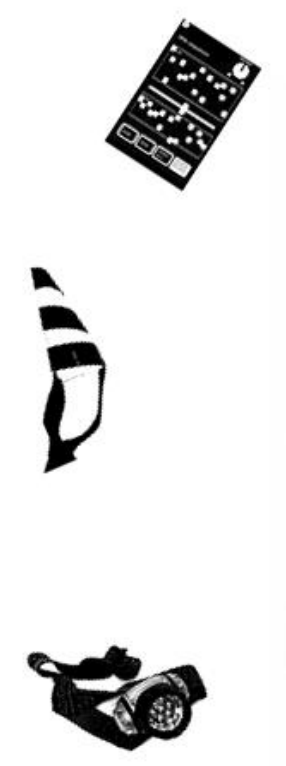

6
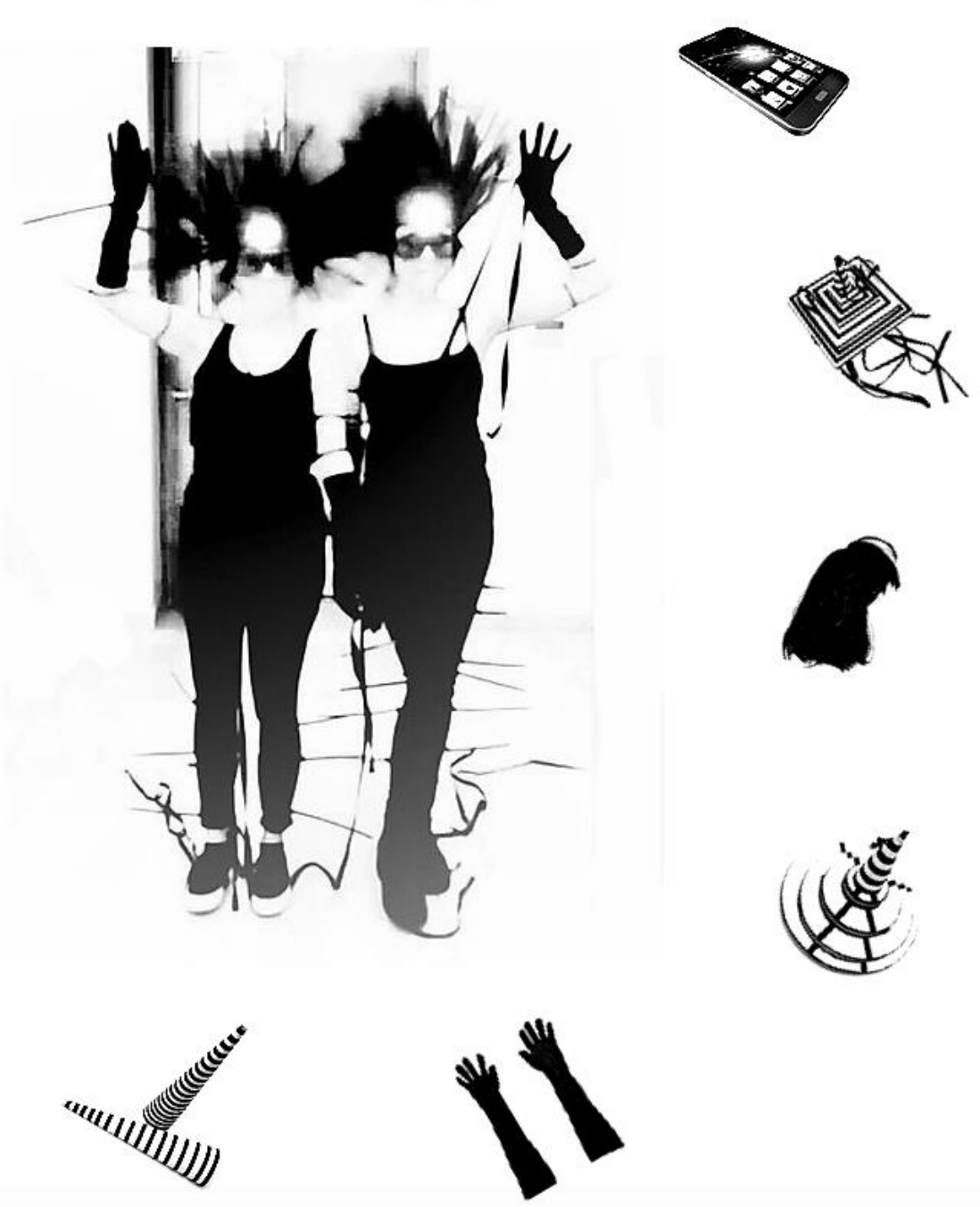

NoDOS(3) (2020). Cabecera de la página. Fuente: las autoras

https://www.thespectacularhouse.com/nodos-3 
Revista de Estudios en Sociedad, Artes y Gestión Cultural
Monográfico Extraordinario II

Octubre 2020

Investigación

ISSN: 2340-9096

https://dx.doi.org/10.17561/rtc.extra2.5726

\section{Action\#1}
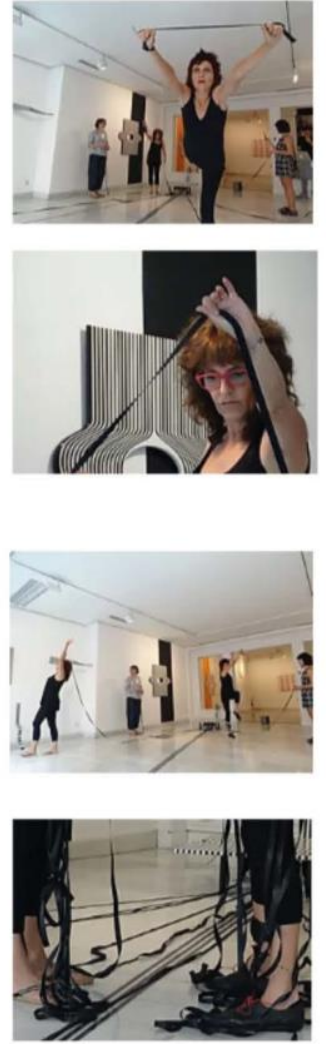

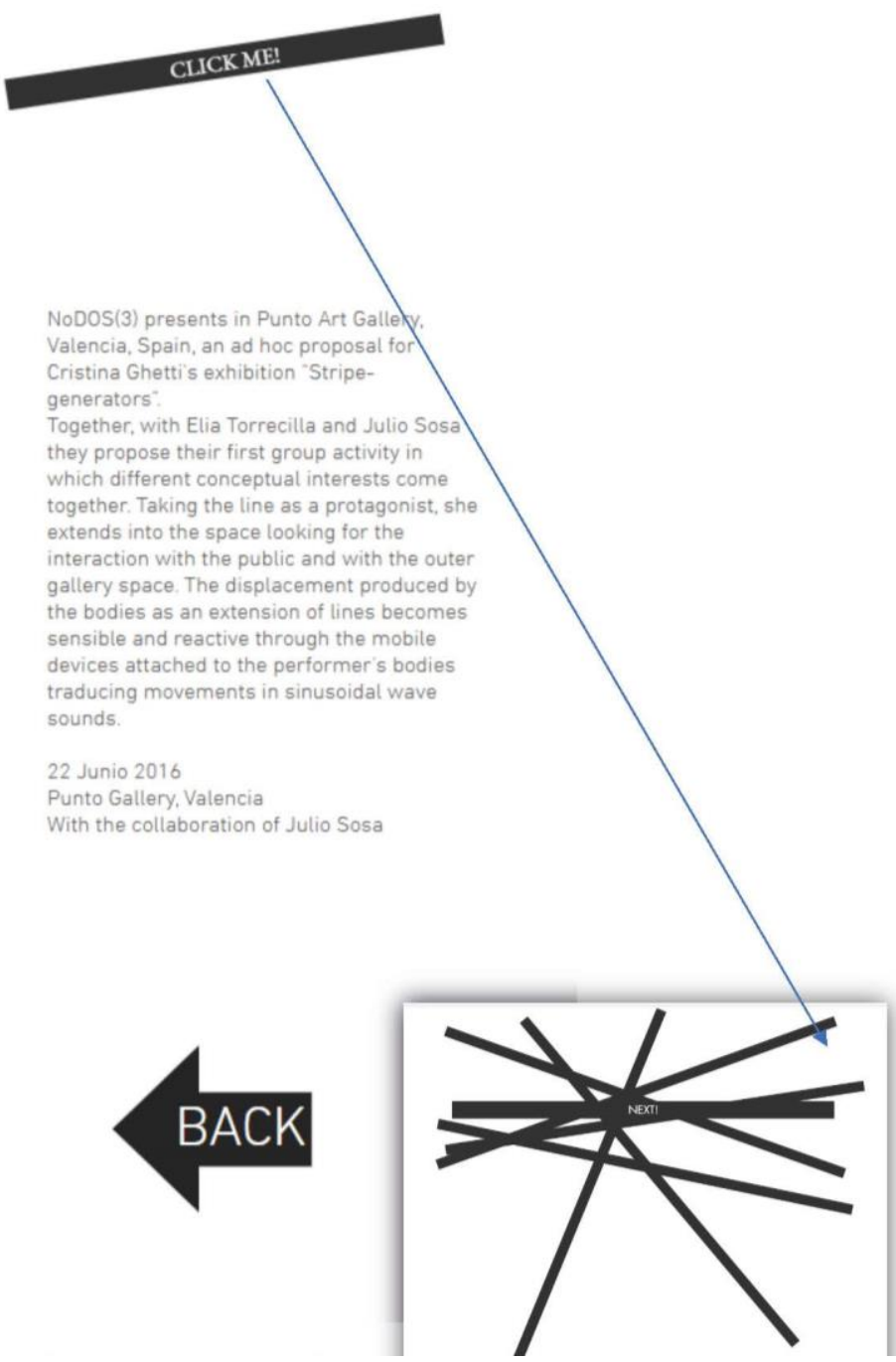

NoDOS(3) (2020). Acción \#1. Fuente: las autoras 
Revista de Estudios en Sociedad, Artes y Gestión Cultural
Monográfico Extraordinario II

Octubre 2020

Investigación

ISSN: $2340-9096$

https://dx.doi.org/10.17561/rtc.extra2.5726

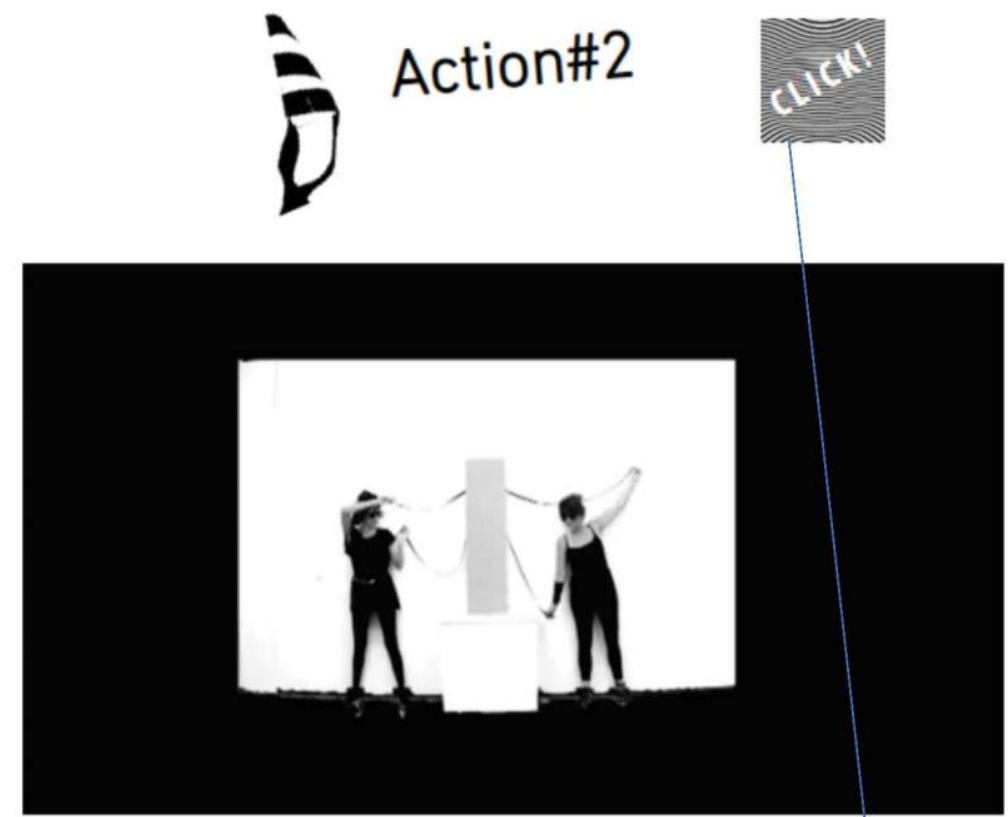

https://www.voutube.com/watch?time continue=283\&v=rpxNZkliGtl\&feature=emb logo

NoDOS(3)'s second action is a hybrid work of art, an audiovisual performance where the virtual and the disembodied, are linked to the physical realm through the pictorial line that vanishes into a purely digital space. A work of art where glitch aesthetic and the relationship of the body with the line in a $2 \mathrm{D}$ space, acquire a third dimension thanks to light and sound generated in real-time.

The work was committed by the Intramurs international art festival held city and presented in Ca Revolta Teather.

23 October 2016

With the collaboration of Julio Sosa
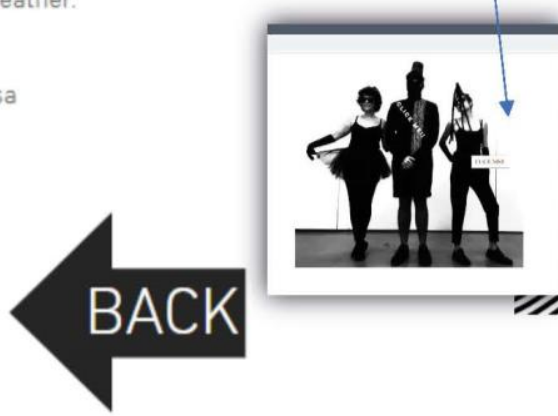
Valencia

NoDOS(3) (2020). Acción \#2. Fuente: las autoras

https://www.thespectacularhouse.com/accion-2 


\section{Tercio Creciente}

ISSN: 2340-9096

https://dx.doi.org/10.17561/rtc.extra2.5726
Monográfico

Extraordinario II

Octubre 2020

Investigación

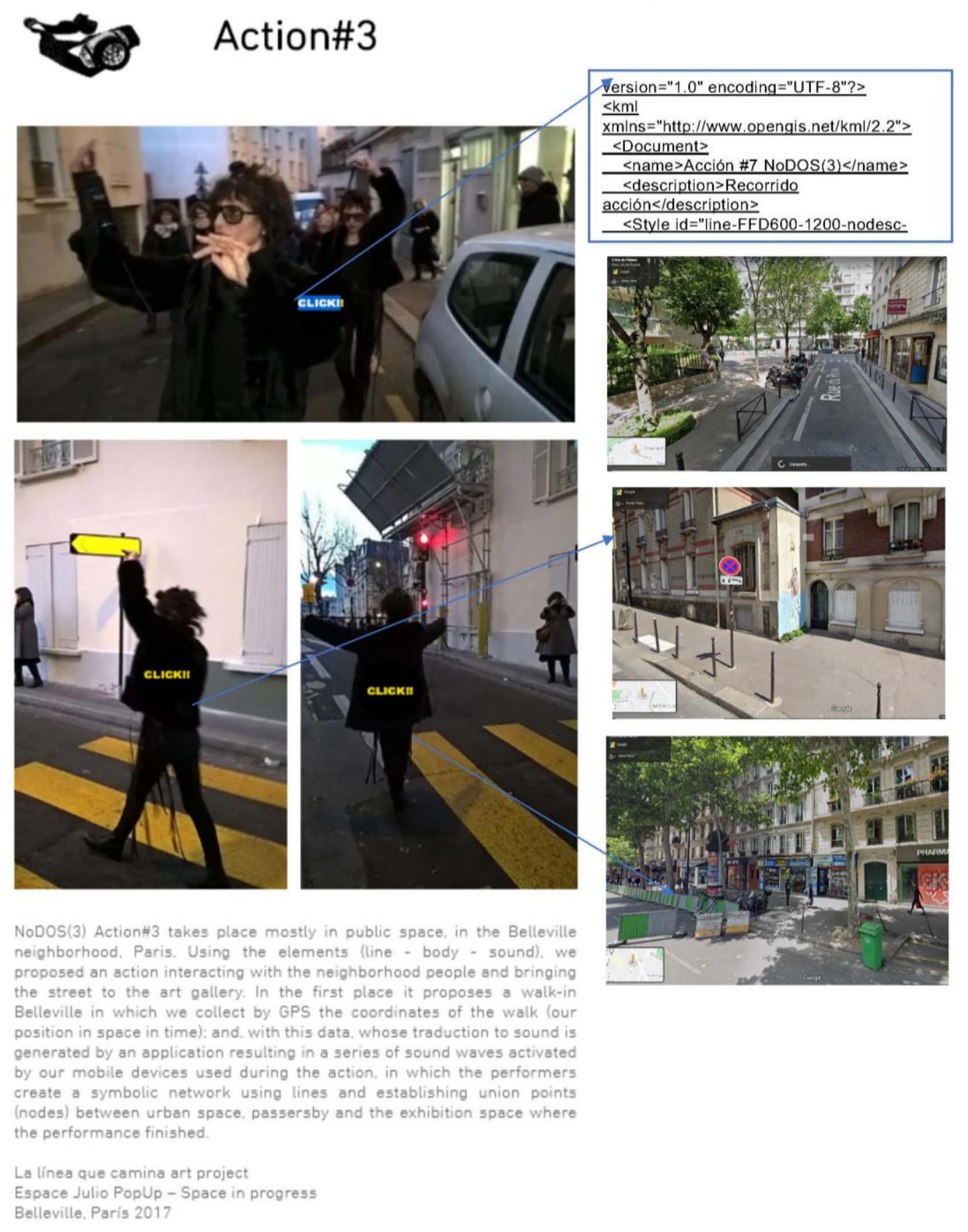

NoDOS(3) (2020). Acción \#3. Fuente: las autoras

https://www.thespectacularhouse.com/accion-3 
Revista de Estudios en Sociedad, Artes y Gestión Cultural

ISSN: 2340-9096

https://dx.doi.org/10.17561/rtc.extra2.5726
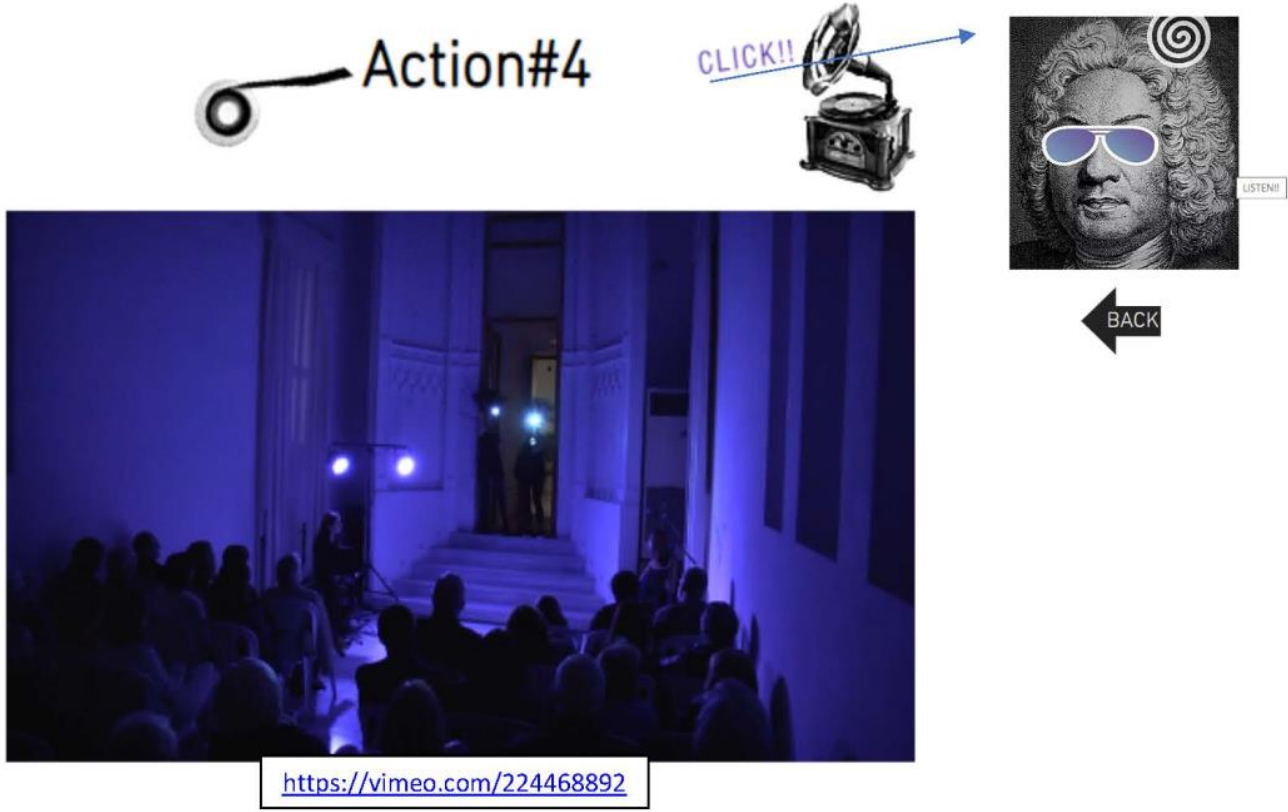

NoDOS(3) Action \#4 The group was invited to perform at the 'Ruta de las Ermitas Hermits routes concerts. organized by the Elche University. No(Dos)3 performed a modified version, manipulating sound with body movements, of "the Barricades Misterieuses" musical piece from Barrocan french musician François Couperin. The ambient of the church, the dressings and the distortions of sound changed the classic piece into a different light sound body movement experience

Villa Gadea Hermit 2017. Altea. Alicante. Spain

With the collaboration of Julio Sosa

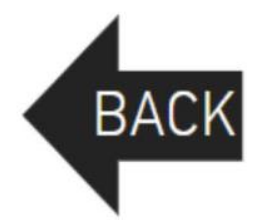

NoDOS(3) (2020). Acción \#4. Fuente: las autoras

https://www.thespectacularhouse.com/accion-4 
Revista de Estudios en Sociedad, Artes y Gestión Cultural
Monográfico Extraordinario II

Octubre 2020

Investigación

ISSN: $2340-9096$

https://dx.doi.org/10.17561/rtc.extra2.5726

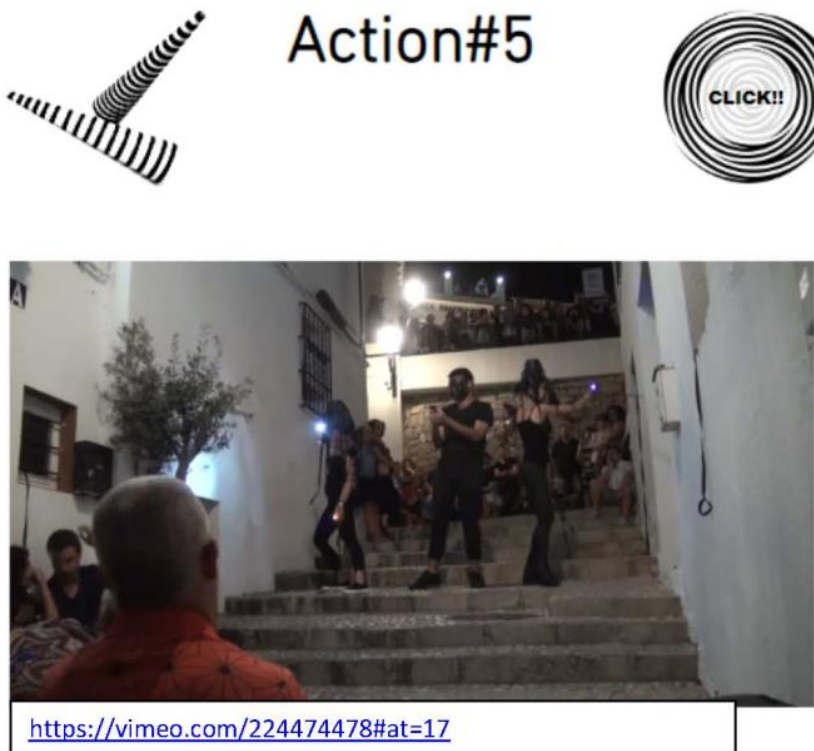

httos://vimeo.con

NoDOS(3) Invited to present a new art piece for the Nit de l'Art Altea (Altea art night) We performed with artist Julio Sosa, interacting with street people in the historic center of Altea Village, the group walked and sonorized a street, appearing surprisingly in the public space, tracing spatial diagrams with lines, as measuring the place for some unknown reason, generating sound with their mobile devices and vanishing into passersby at the end.

The historic center of Altea Village (public action) 2017
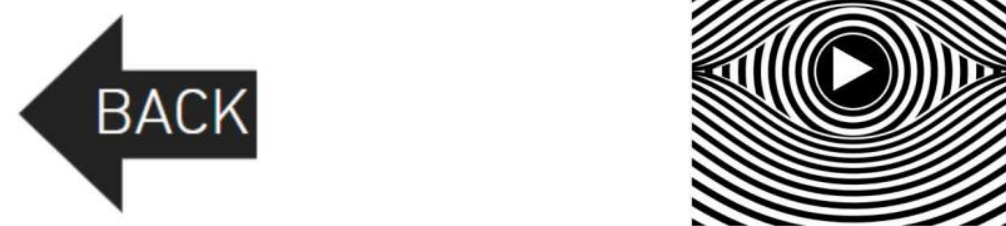

NoDOS(3) (2020). Acción \#5. Fuente: las autoras

https://www.thespectacularhouse.com/accion-5 
Revista de Estudios en Sociedad, Artes y Gestión Cultural

ISSN: 2340-9096

https://dx.doi.org/10.17561/rtc.extra2.5726

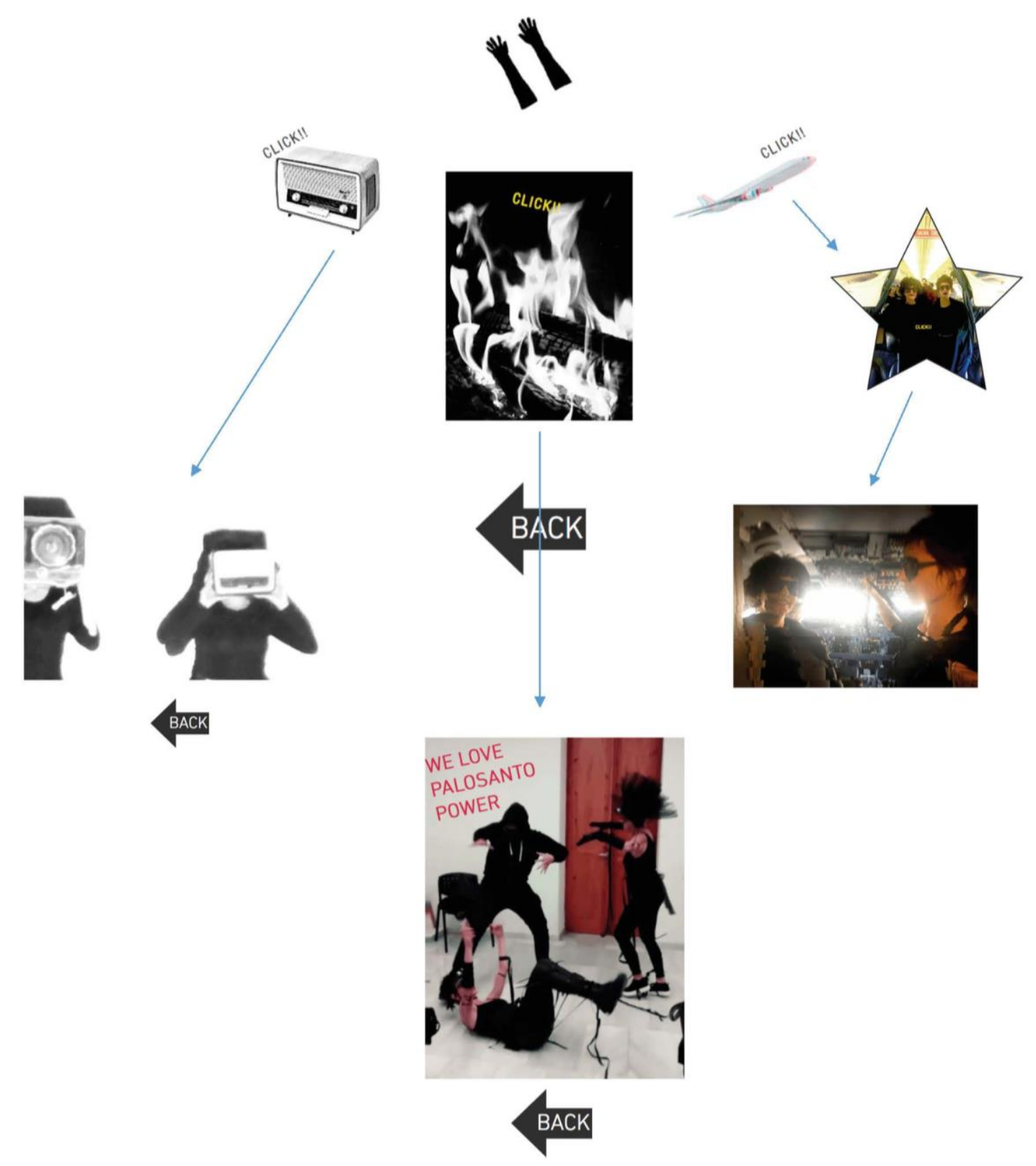

NoDOS(3) (2020). Bonus track. Fuente: las autoras

https://www.thespectacularhouse.com/accion-6 
Revista de Estudios en Sociedad, Artes y Gestión Cultural
Monográfico Extraordinario II

Octubre 2020

Investigación

ISSN: 2340-9096

https://dx.doi.org/10.17561/rtc.extra2.5726

\section{Action\#7}
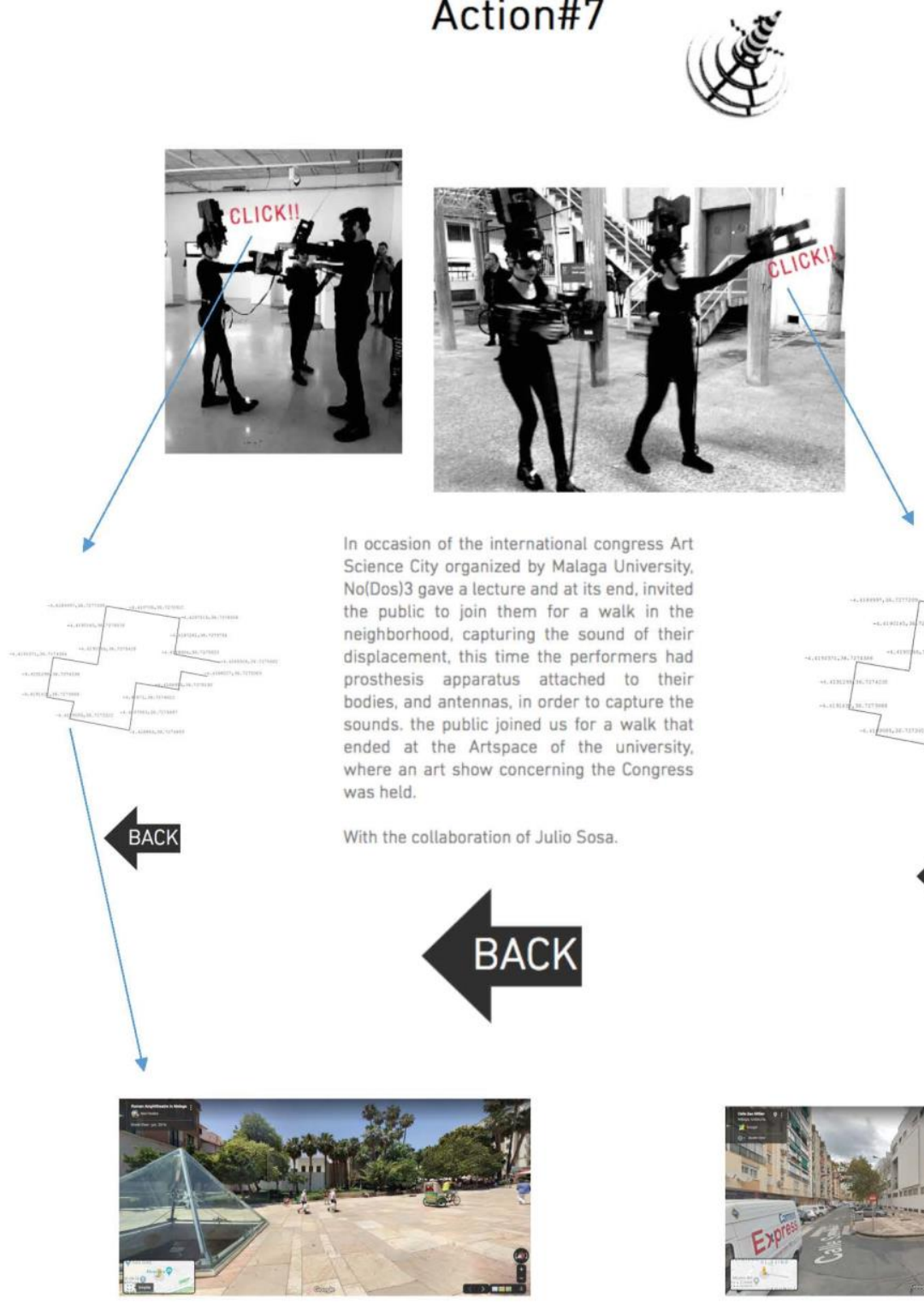 the had their that .
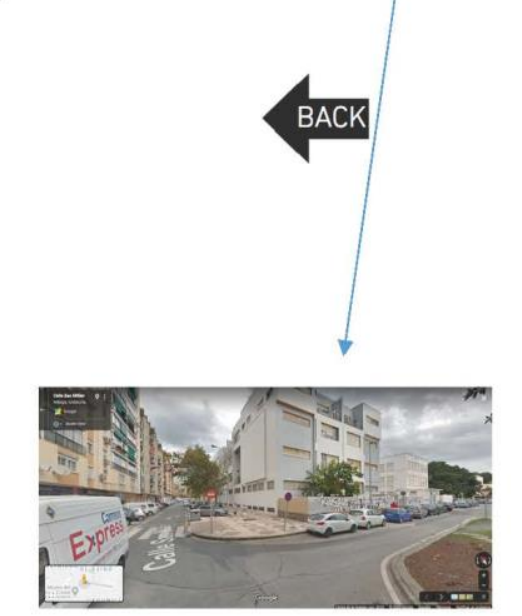

NoDOS(3) (2020). Acción \#7. Fuente: las autoras

https://www.thespectacularhouse.com/accion-7 
Revista de Estudios en Sociedad, Artes y Gestión Cultural

ISSN: $2340-9096$

https://dx.doi.org/10.17561/rtc.extra2.5726 raordinario II

Octubre 2020

Investigación

\section{Action\#8}
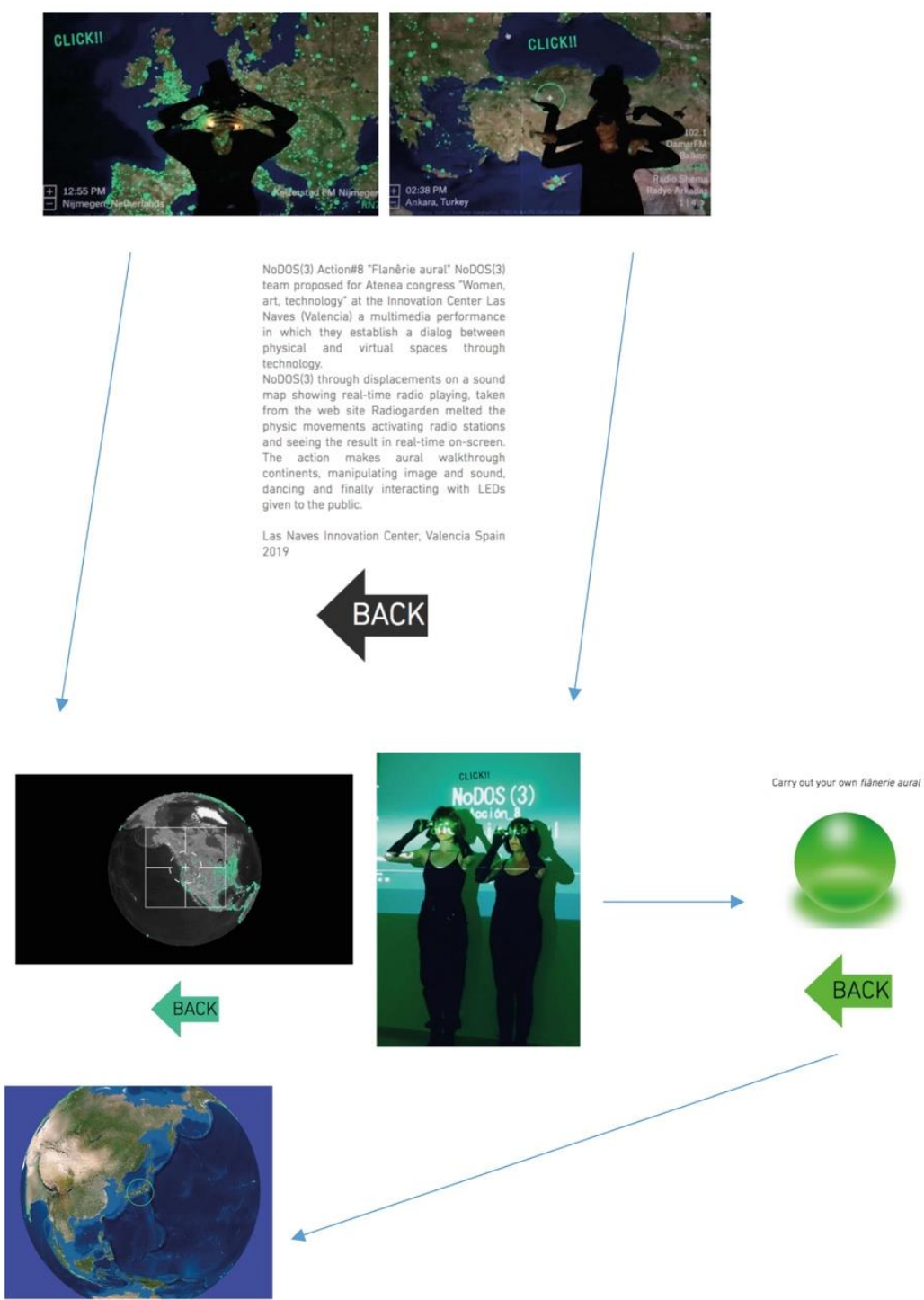

NoDOS(3) (2020). Acción \#8. Fuente: las autoras

https://www.thespectacularhouse.com/accion-8 
Revista de Estudios en Sociedad, Artes y Gestión Cultural

Monográfico Extraordinario II

Octubre 2020

Investigación

ISSN: 2340-9096

https://dx.doi.org/10.17561/rtc.extra2.5726

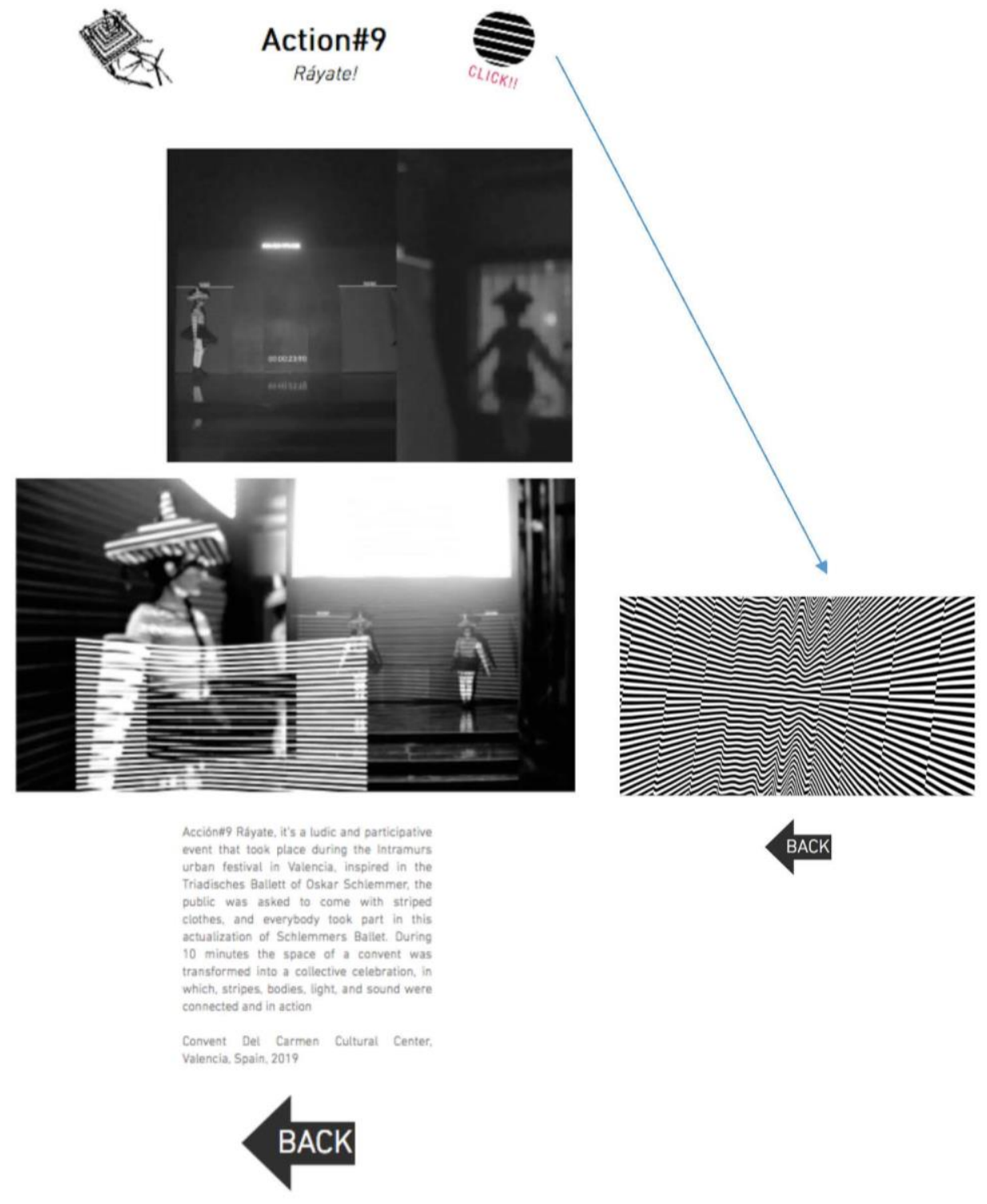

NoDOS(3) (2020). Acción \#9. Fuente: las autoras

https://www.thespectacularhouse.com/accion-9 
Revista de Estudios en Sociedad, Artes y Gestión Cultural
Monográfico Extraordinario II

Octubre 2020

Investigación

ISSN: 2340-9096

https://dx.doi.org/10.17561/rtc.extra2.5726

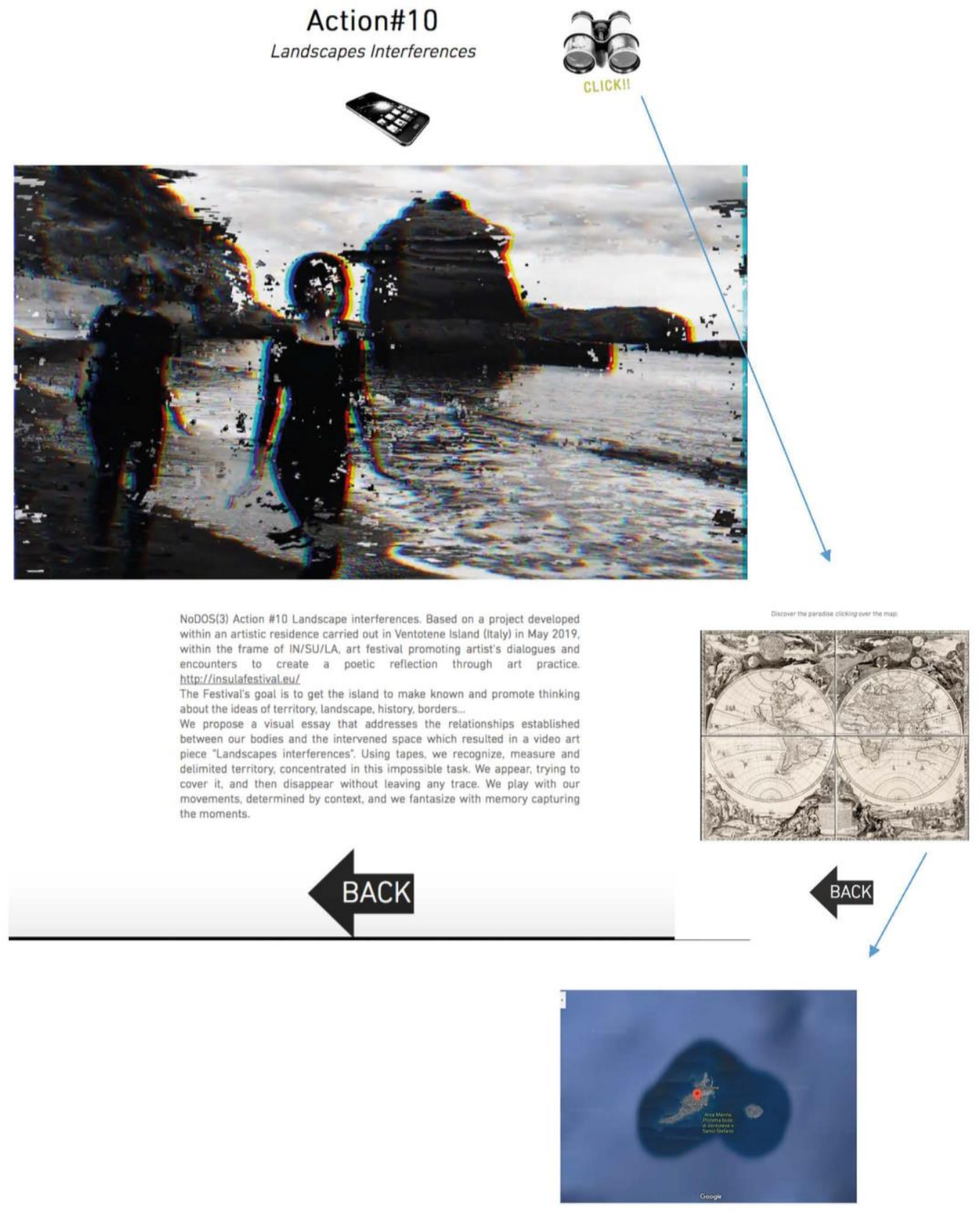

NoDOS(3) (2020). Acción \#10. Fuente: las autoras

https://www.thespectacularhouse.com/accion-10 


\section{Conclusión}

El trabajo de $\operatorname{NoDOS(3)~es~de~naturaleza~híbrida.~Esta~experiencia~nos~ha~permitido~la~}$ posibilidad de una visualización, también híbrida, de nuestras acciones en el campo de la realidad.

La experiencia se cerró con un encuentro virtual online donde pudimos responder a preguntas de nuestros usuarios y obtener un feedback de nuestro trabajo.

Cabe decir, que esta experiencia fue desarrollada en periodo de confinamiento y que la esencia virtual de esta residencia cobró un significado más potente aún dadas las circunstancias.

Ello permitió que personas de todo el mundo, desde sus casas, pudieran conocer nuestro trabajo, y a nosotras poder realizar un trabajo vía telemática sin la necesidad de desplazarnos a un espacio físico cuyo acceso estaba limitado en esos momentos.

\section{Referencias}

Torrecilla, Elia; Ghetti, Cristina. Interferencias en el paisaje. Cuerpo y espacio en la práctica artística de NoDOS(3). ANIAV - Revista de Investigación en Artes Visuales, [S.1.], n. 6, p. 95-107, mar. 2020. https://doi.org/10.4995/aniav.2020.12948

Torrecilla, Elia. (2018). "El lenguaje de las interfaces en movimiento: La hibridación en la práctica artística de NoDOS (3)". ASRI. Arte y Sociedad. Revista de Investigación, (15), 714.

Torrecilla, E. (2018). Flâneur, Ciberflâneur, Phoneur: Un paseo de resignificación del

Flaneur por el espacio urbano moderno, el ciberespacio y el espacio híbrido.

Acciones artísticas propias entre 2011-2018 [Tesis doctoral]. Universitat Politècnica

De València. Disponible en: https://riunet.upv.es/handle/10251/107391 
Revista de Estudios en Sociedad Artes y Gestión Cultural

ISSN: 2340-9096

https://dx.doi.org/10.17561/rtc.extra2.5726
Monográfico

154

xtraordinario II

Octubre 2020

Investigación 